\title{
The role of physiotherapist after breast cancer surgery
}

\section{Abstract}

Several physical impairments are developed after breast cancer treatments (surgery, radiation, chemotherapy), commonly found lymphedema, frozen shoulder, cancerrelated fatigue, axillary web syndrome and increased weight. Physiotherapists have an essential role on the management of these consequences; they certainly could make a difference in the quality of life of survivors; Education from physiotherapist to patients about these possible impairments pre and post $\mathrm{BC}$ treatments is the primary key for them to seek early diagnose and manage.

Objective: to describe the most common physical impairments that patients may develop after breast cancer surgery and how physiotherapists could address these to increase the quality of life in these patients.
Volume 2 Issue 6 - 2018

\section{Alejandra Marissa Fernandez Reyes}

Universidad del Futbol y Ciencias del deporte, Pachuca, Mexico

Correspondence: Alejandra Marissa Fernandez Reyes, Universidad del Futbol y Ciencias del Deporte, Libramiento circuito de la Concepcion Sur n, La Concepcion, 42160 San Agustin Tlaxcala, Hgo, México, Tel 6865450377.

Emailmarijssaf_r@hotmail.com

Received: October 21, 2018 | Published: December 17, 2018

Keywords: breast cancer, mastectomy, lymphedema, physiotherapist, complete decongestive therapy, axillary web syndrome, exercise after breast cancer

Study selection: selected articles related to breast cancer physical impairments after surgery and physiotherapy procedures to manage them were assessed in PubMed database during 2018.

\section{Introduction}

\section{Data synthesis:}

\section{Lymphedema: ${ }^{1-4}$}

Definition: Accumulation of protein-rich fluid in interstitial spaces due to impairment of the lymphatic system. There are described two types of lymphedema: primary and secondary. Primary is related to lymphatic system malformation and some genetic conditions. Secondary Lymphedema is related to damage to lymphatic system secondary to other procedures as surgeries, traumatism, infections, cancer etc. This condition is often under-diagnosed, and there is a massive lack of education between patients and health professionals about how to address it.

\section{Secondary lymphedema due to breast cancer surgery symptoms:}

Arm heaviness Figure 1

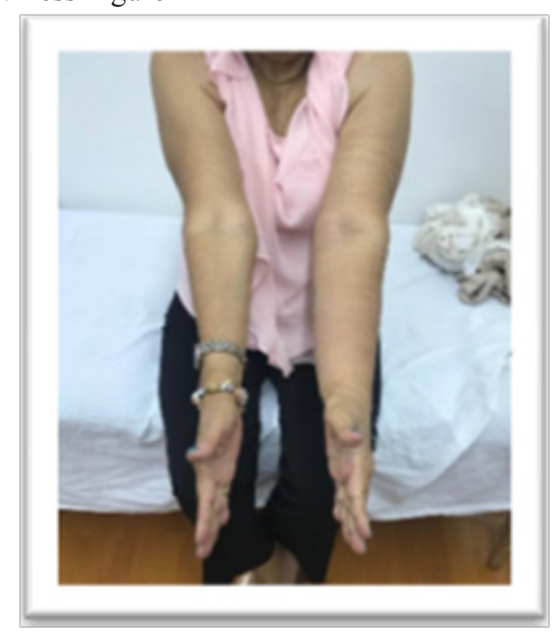

Figure I Secondary lymphedema due to breast cancer surgery symptoms.
I. Paresthesia

II. Arm ,breast and or upper trunk increased volume

III. Diminished range of motion

IV. Infections (cellulitis)

V. Tissue changes ( fibrosis, cysts, fistulas, , etc)

\section{Diagnosis: ${ }^{1}$}

Arm measurements: circumferential measurements should be made every $4 \mathrm{~cm}$ starting from MTCF joints and $2 \mathrm{~cm}$ above radial epiphysis.

I. "The $2 \mathrm{~cm}$ rule": when $2 \mathrm{~cm}$ circumferential different (limb discrepancy at one point) has been found intensive treatment must be started.

II. Photographies

III. Volumetry (sum of the perimeter at every 4 points divided into 3.14)

IV. Palpations (godet and stemmer sign to evaluate fibrotic tissue)

V. Observation (skin folds, infection, skin color, etc)

VI. Imagenology: Lymphoscintigraphy, lymphography, ultrasound, MRI, magnetic bioimpedance.

Treatment: Standard gold treatment internationally recognized is known as complex decongestive therapy (CDT), it consists of 4 steps applied in 2 phases and continuous outcomes evaluation and self-education for affected patients Table 1.

\section{CDT Methods (brief description) ${ }^{1-4}$}

Manual lymph drainage: Described as a gentle manual treatment technique based on "Vodder strokes." It as a working phase where stretch stimuli are applied to the subcutaneous tissues with the objective of rerouting fluid from"blocked" áreas into healthy lymph vessels; And a resting phase where this pressure is released promoting absorption of fluid from interstitial spaces. The pressure applied is 
light, should not produce active hyperemia (vasodilatation) or damage to the lymphatic structures, although more pressure is needed if fibrotic tissue is involved.
General effects of MLD: Increase in lymph production; increase in lymphangionmotoricity; reverse of lymph Flow; increase in venous return; analgesic effect:

Table I Lymphedema stages (International Society of Lymphology)

\begin{tabular}{llll}
\hline Stage 0 & Stage I & Stage II & Stage III \\
\hline Subclinical stage & Early stage & Fibrotic tissue + & Hard fibrotic tissue + \\
\hline No evidente swelling & Fluid accumulation(pitting +$)$ & Pitting can be or not present & Absent pitting \\
$\begin{array}{l}\text { Impaired lymph } \\
\text { transport. }\end{array}$ & Fluid subsides with elevation & $\begin{array}{l}\text { Elevation rarely decrease } \\
\text { fluid }\end{array}$ & $\begin{array}{l}\text { Skin changes: skinfolds, papilomas,fat } \\
\text { deposits, cyst etc. }\end{array}$ \\
\hline
\end{tabular}

\section{Compression}

External support of the affected lymph is essential for the management of lymphedema, its primary goal is to maintain the decongestive effect achieved in MLD treatments, therefore prevents from reaccumulation of fluid into the tissues. In intensive phase, compression is applied by "short stretch bandage" with the objective to achieve a maximal reduction of limb volume. In this technique, the physiotherapist creates a compression gradient with several bandage layers and methods, to obtain more pressure in distal areas and less pressure in proximal áreas, this Will promote the reabsorption of interstitial fluid and reverse of lymph fluid from distal to proximal and healthy structures. In the maintenance phase, compression is applied with compression garments as arm sleeve, gloves and or gauntlets to maintain volume reduction and prevent further complications; garments should be wear throughout a patient's life. Garments should be fitted by a certified practitioner who is in charge of select the adequate compression grade, brand and materials for every case. General effects of compression: increase tissue pressure, promoting beneficial effects for Exchange of fluids between capillaries and tissues, improves venous and lymphatic return, reduce net filtration, improves muscle pump during activity, prevents reaccumulation of fluid, helps to break up fibrotic tissue, provides support to tissues Figure 2.

\section{Decongestive exercise (remedial exercise)}

Exercise performed during intensive phase is called "remedial exercise," It should be done under the supervision of a physiotherapist and always wearing compression bandage to assist the body in remodeling tissue and returning lymphatic fluid to the circulatory system. There is some controversy about type and dose of exercise for lymphedema patients, although several programs include: resistance training, aerobic training, and breathing exercise. One prospective study comprised of resistance band exercises 5 times a week for 8 weeks were initially supervised during the intensive lymphedema treatment, but performed independently during the study period, after 8 weeks, the intervention group demonstrated statistically significant differences, upper body resistance exercise shows a positive effect on arm function and muscular strength without increasing arm volume in breast cancer-related lymphedema. ${ }^{8}$

Skincare: Patients with lymphedema are at risk of developing infections of skin and nails, meticulous care is essential for the success of CDT. The physiotherapist should instruct patients how to clean and moisturize these areas properly, and how to evaluate and maintain their integrity.
TDC objective (1,4): These methods are applied in two phases, as mentioned above; The physiotherapist implements intensive period for at least 2-4 weeks of daily treatment and maintenance phase is achieved mainly by self-treatment after the physiotherapist educate the patient enough to manage their lymphedema, this phase is maintained during whole patient's life. CDT provides enhancement of upper extremity functions, reduction in lymphedema volume, pain, heaviness and the improvement in shoulder mobility. ${ }^{5}$

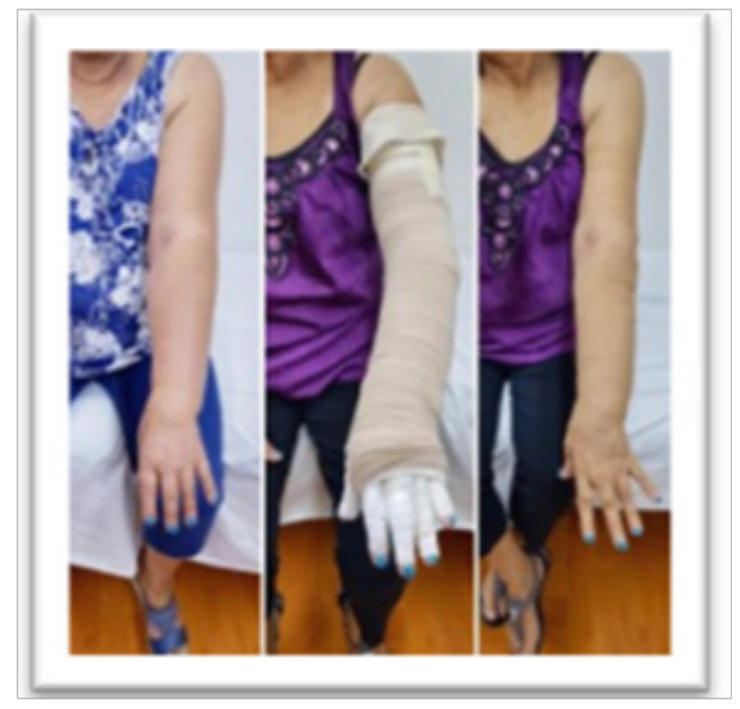

Figure 2 Exchange of fluids between capillaries and tissues, improves venous and lymphatic return, reduce net filtration, improves muscle pump during activity, prevents reaccumulation of fluid, helps to break up fibrotic tissue, provides support to tissues.

Axillary web syndrome: One article was found related to "axillary web syndrome" (AWS), also known as "cording," a common sequelae after mastectomy but often undiagnosed and untreated. The first symptoms consist in "cord a-like" structure beneath the axilla, especially visible during arm abduction, it may disappear by itself after a few weeks, but in some cases, it needs further treatment to avoid impairment in shoulder movement and function. These treatments consist of passive, active and resisted movements guided by a physiotherapist; it seems that the best intervention for this entity it is early patient education before and after breast cancer surgery, focusing in explaining correct time frame for initiation of gentle passive ROM and adequate dose and periodization of strength training. ${ }^{6}$ 


\section{Cancer related fatigue, weight management and exercise (6)}

Cancer-related fatigue is defined as a distressing and persistent sense of tiredness or exhaustion that is related to cancer or its treatment, it is reported in $50-70 \%$ of patients undergoing treatment, and survivors report lasting symptoms for months to years after finishing treatment. The National Comprehensive Cancer Network (NCCN) published guidelines to evaluate cancer-related fatigue. Initial assessment includes emotional distress, pain, sleep disturbance, medication side effects, hypothyroidism, and anemia. If none of this factors are present or they persist despite treatment, nonpharmacological interventions are recommended, they include activity enhancement with exercise programs, psychological interventions for stress and anxiety, nutrition counseling and sleep therapy. Body mass index has been examined considerably as a hypothesized prognosticator for a breast cancer survivor. Most studies associate overweight and obesity pre and post-treatment with an increased risk of breast cancer recurrence and shortened survival times. All types of activity are beneficial, with some stronger effects for recreational activity. At least four hours of exercise per week of moderate to vigorous intensity program is necessary to reduce risk significantly. ${ }^{7,8}$

\section{Conclusion}

Breast cancer survivors continuously struggle with several physical and mental consequences after breast cancer treatments. These consequences are not being diagnosed or treated as often as they require. The physiotherapist must become aware of its essential role in this consequences and how they could offer an adequate treatment, become an active educator within patients and health care professionals in order to improve patients quality of life and their ability for self-management. It seems that raising patients' awareness and training healthcare professionals regarding lymphedema preventive strategies have an important role in earlier and better combating this complication Figure 3. ${ }^{9}$

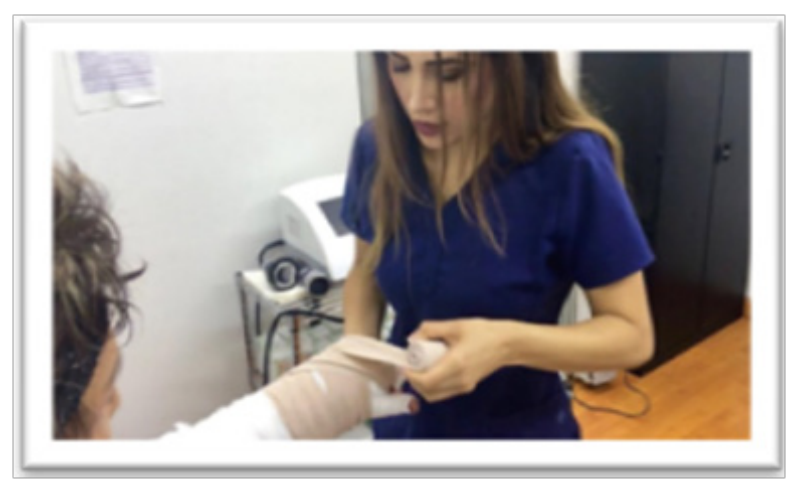

Figure 3 Training healthcare professionals regarding lymphedema preventive strategies have an important role in earlier and better combating this complication.

\section{Acknowledgments}

None.

\section{Conflicts of interest}

The author declares there is no conflict of interest.

\section{References}

1. Zuther JE, Norton S. Lymphedema Managment, the Comprehensive Guide for Practitioners. $3^{\text {rd }}$ edition. Stuttgart, Germany: Thieme; 2013;129-161.

2. Ezzo J, Manheimer E, McNeely ML, et al. Manual lymphatic drainage for lymphedema following breast cancer treatment. Cochrane Database Syst Rev. 2015;21(5):CD003475.

3. Moeller J, Cleveland S, Schaverien M. Nonoperative Treatment of Lymphedema. Seminars in Plastic Surgery. 2018;32(01):17-021.

4. Cho Y, Do J, Jung S, et al. Effects of a physical therapy program combined with manual lymphatic drainage on shoulder function, quality of life, lymphedema incidence, and pain in breast cancer patients with axillary web syndrome following axillary dissection. Supportive Care in Cancer. 2015;24(5):2047-2057.

5. Ozcan DS, Dalyan M, Delialioglu SU, et al. Complex Decongestive Therapy Enhances Upper Limb Functions in Patients with Breast Cancer-Related Lymphedema. Lymphatic Research and Biology. 2018;16(5):446-452.

6. Harris SR. Axillary Web Syndrome in Breast Cancer: A Prevalent But Under-Recognized Postoperative Complication. Breast Care. 2018;13(2):132-135

7. Irwin ML. ACSM's Guide to Exercise and Cancer Survivorship. 2nd edition. Human Kinetics; 2012. p. 16-25.

8. Do JH. Effects of resistance exercises and complex decongestive therapy on arm fucntion and muscular strenght in breast caancer-related lymphedema. Lymphology. 2015;48(4):184-196.

9. Oshnari LA, Hosseini SA, Haghighat S, et al. The Effect of Complete Decongestive Therapy on Edema Volume Reduction and Pain in Women With Post Breast Surgery Lymph Edema. Iranian Journal of Cancer Prevention. 2016;9(2):e4209. 